\title{
Position Dependent Resistance and Doping Condition on a Graphene Flake
}

Received September 2, 2020; revised September 20, 2020; accepted October 10, 2020

Oh Hun Gwon ${ }^{a}$, Jong Yun Kim ${ }^{b}$, Seok-Ju Kang ${ }^{b}$, and Young-Jun Yu ${ }^{a, b *}$

aDepartment of Physics, Chungnam National University, Daejeon 34134, Republic of Korea

bInstitute of Quantum Systems, Chungnam National University, Daejeon 34134, Republic of Korea

*Corresponding author E-mail: yjyu@cnu.ac.kr

\begin{abstract}
In this work, we investigated the variation in surface conditions of graphene by employing atomic force microscopy and optical images. We inspected the resistance and doping at selected areas of graphene by transport measurements. Especially, the area with a dominant contribution from the edges, which have more disorders and poly (methyl methacrylate) residues compared to the central surface of graphene, showed large resistance as well as large doping concentration. Our study facilitated the understanding of the electric conditions on graphene surface and edge areas.
\end{abstract}

Keywords: Graphene, Carrier density, Resistance, Transport characterization

\section{Introduction}

Graphene, a two-dimensional carbon atom network, has been studied for applications in transparent and flexible electrodes. In particular, the formation of high-quality and large-size graphene with perfect carbon crystal is one of the major goals in research involving graphene [1-7]. Although the electric property of a graphene flake is characterized by measuring resistance and accumulated carrier density, these values are averaged over areas with varying condition such as disorders, and residues on graphene flake. Thus, we should understand the role of disordered areas on graphene flake. Several studies have carried out the spatial investigation of graphene surface by employing a scanning probe microscope (SPM) $[8,9,12]$. However, since the characterization using SPM has limitations for inspecting the direct conductance of graphene, electron transport characterization is preferred for direct measurement of the conductance of graphene [8-12]. In this work, we study the electric properties of partial areas of graphene, primarily focusing on either the center or the edge, by electron transport characterization. We observe that the graphene channel with a large contribution from the edge area shows relatively high resistance and increased p-doping level, due to more disorders and Poly(methyl methacrylate) (PMMA) residues.

\section{Experimental details}

For measuring the resistance, graphene flakes were mechanically exfoliated on a 280 -nm-thick $\mathrm{SiO}_{2}$ substrate and $\mathrm{Cr} / \mathrm{Au}(5 \mathrm{~nm} / 30 \mathrm{~nm}$ thickness) electrodes were used to make electric contacts, as shown in Fig. 1(a) [4-7]. For making the contacts with $\mathrm{Cr} / \mathrm{Au}$ electrodes, PMMA was employed as a mask in the e-beam lithography process and this PMMA was removed by acetone solution after evaporating $\mathrm{Cr} / \mathrm{Au}$ metal. Each thickness of graphene was confirmed by inspection using a commercial atomic force microscope (AFM, XE-100, Park systems Corp.) $[8,9,12]$. The resistance of graphene as a function of back-gate voltage $\left(V_{\mathrm{BG}}\right)$ through the $\mathrm{SiO}_{2}$ substrate was measured by the 2-terminal electron transport measurement [8-12].

\section{Results and discussion}

Figures 1(b) and 1(c) show the AFM image and height profile between $\mathrm{SiO}_{2}$ and graphene, respectively. Here, the graphene is confirmed as a bilayer with a height difference of $\sim 0.6 \mathrm{~nm}$ between graphene and $\mathrm{SiO}_{2}$ [see Fig. 1(c)]. On this clean surface of bilayer graphene, the charge-neutral position $\left(V_{\mathrm{CPD}}\right)$ was obtained at $V_{\mathrm{CPD}} \sim$ $74 \mathrm{~V}$ with $R \sim 8.28 \mathrm{k} \Omega$ that corresponds to the highest position (marked
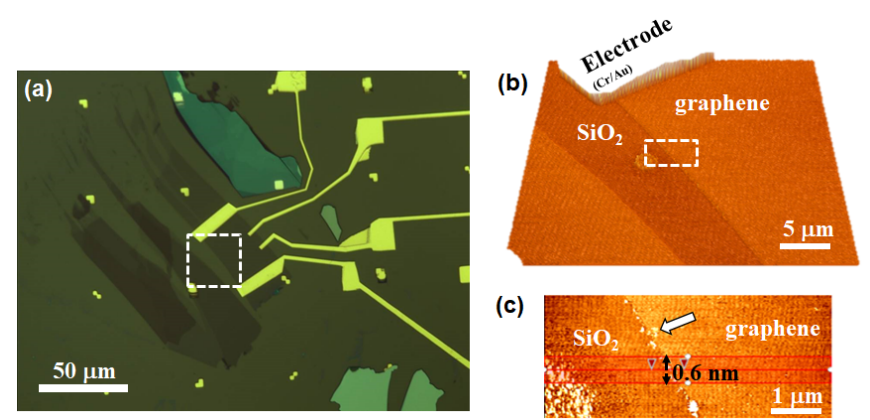

(c)

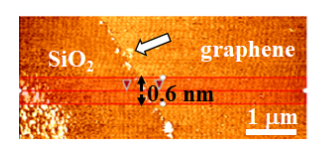

Figure 1. (a) Optical image of graphene field effect transistor (FET) with $\mathrm{Cr} / \mathrm{Au}$ electrodes contacts on the $\mathrm{SiO}_{2}$ substrate. (b) Topography image of the dashed box shown in (a). (c) Enlarged topography image of the dashed box in (b), the height of bi-layer graphene and residues are marked by black and white arrows, respectively. 


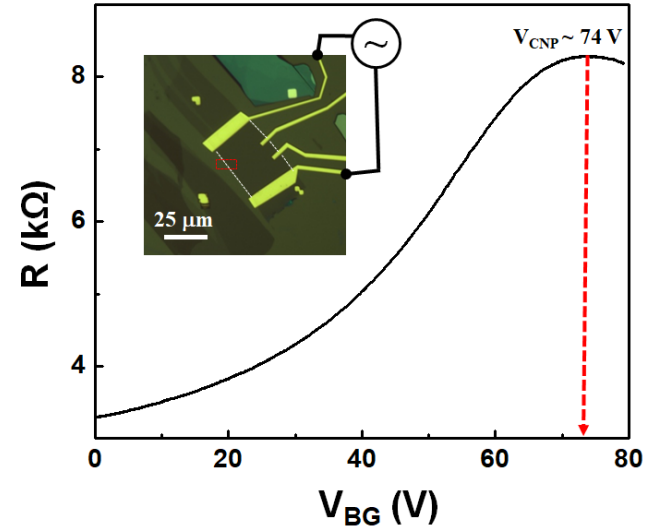

Figure 2. Resistance of bi-layer graphene as a function of back-gate voltage $V_{B G}$. Inset: Optical image of bi-layer graphene with a schematic image of a conductance measurement.
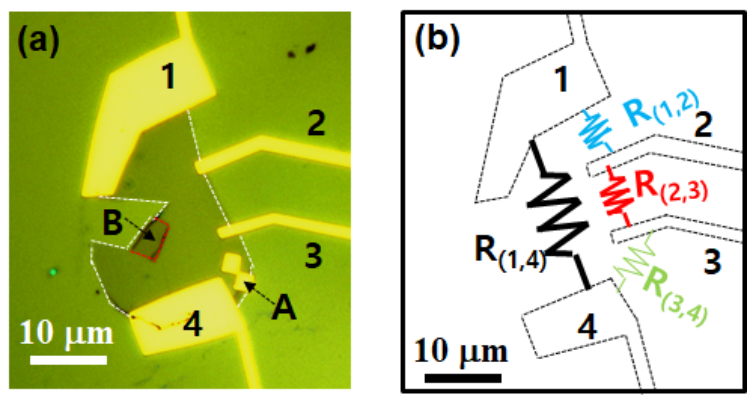

Figure 3. (a) Optical image of graphene FET with $\mathrm{Cr} / \mathrm{Au}$ electrodes on the $\mathrm{SiO}_{2}$ substrate. (b) The schematic diagram for $R_{(i, j)}$ between $\mathrm{i}$ and $\mathrm{j}$-electrodes.

with dashed red arrow) in the transport curve plotted in Fig. 2. This bilayer is heavily p-doped with $n=\left(C_{\mathrm{BG}} / e\right) \cdot V_{\mathrm{CNP}} \approx 5.18 \times 10^{12} \mathrm{~cm}^{-2}$, where $C_{\mathrm{BG}}=12 \mathrm{nF} / \mathrm{cm}^{2}, e$, and $V_{\mathrm{CNP}}$ represent capacitance for $280-\mathrm{nm}$ thick $\mathrm{SiO}_{2}$, the charge of an electron, and charge-neutral position of this bilayer graphene, respectively [8-12]. While disorders are not observed on the graphene surface, undesired residues attached to the edge of the bilayer graphene were found [marked with an arrow in Fig. 1(c)]. Generally, these residues are PMMA polymers, which are not removed during fabrication process for metal electrodes contacts and lead to hole doping in graphene. Since the bonds are terminated at the crystal edge, it contains more defects. Thus, PMMA residues are strongly bonded at the edges compared to center of the surface, which contains less amount of disorders. In order to understand partial electrical condition difference on the graphene flake, we employed one more graphene flake, which intentionally contains the undesired disorders as shown in Fig. 3. Figure 3(a) shows the optical microscope image of the graphene flake where the $\mathrm{Cr} / \mathrm{Au}$ metal deposited area (marked with A) and the folded area (marked with B) are visible. Since $\mathrm{Cr} / \mathrm{Au}$ electrodes contacts were also made on this graphene flake, there should be unremoved PMMA residues in the disordered region and the edges. Note that, if we had employed the hightemperature annealing process for removing PMMA residues during the device fabrication process, investigation of device performance under the influence of only the edge disorders would have been possible. To study the electrical properties of the partial area of the disordered graphene flake, we measured transport curves as a function
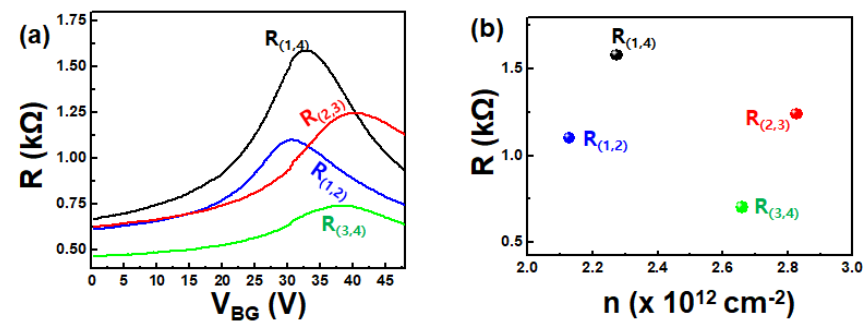

Figure 4. (a) Resistances $R_{(\mathrm{i}, \mathrm{j})}$ measured between $\mathrm{i}$ and j-electrodes as a function of $V_{\mathrm{BG}}$. Here $\mathrm{i}$ and j are $1,2,3$, and 4 , and $\mathrm{i} \neq \mathrm{j}$. (b) Resistance $R_{(\mathrm{i}, \mathrm{j})}$ vs. accumulated carrier density $n$.

of $V_{\mathrm{BG}}$ via various combinations of electrodes. Connections between electrodes 1 and 4,1 and 2, 2 and 3, and 3 and 4 [number of electrodes marked in Fig. 3(a)], and the resistances $\left(R_{(\mathrm{i}, \mathrm{j})}\right)$ between the electrodes on the graphene flake are illustrated with Fig. $3(\mathrm{~b})$.

As a result, we observed various transport curves with different resistance $(R)$ and $V_{\mathrm{CNP}}$ as shown in Fig. 4(a). The $R$ and $V_{\mathrm{CNP}}$ for each electrode combination were found to be $R_{(1,4)}=1.58 \mathrm{k} \Omega$ and $V_{\mathrm{CNP}(1,4)}$ $=32.5 \mathrm{~V} ; R_{(1,2)}=1.1 \mathrm{k} \Omega$ and $V_{\mathrm{CNP}(1,2)}=30.4 \mathrm{~V} ; R_{(2,3)}=1.24 \mathrm{k} \Omega$ and $V_{\mathrm{CNP}(2,3)}=40.4 \mathrm{~V}$, and $R_{(3,4)}=0.7 \mathrm{k} \Omega$ and $V_{\mathrm{CNP}(3,4)}=38 \mathrm{~V}$. From the aforementioned values, the accumulated carrier density, $n$, for each $R$ is plotted in Fig. 4(b). The edges and PMMA residues block the current flow and increase the accumulated hole charge carrier density in graphene channels leading to a large $R$ and $V_{\mathrm{CNP}}$, respectively. We inspect the partial electric property variations by employing the partial values of $R_{(\mathrm{i}, \mathrm{j})}$, and $V_{\mathrm{CNP}(\mathrm{i}, \mathrm{j}) \text {. }}$

By comparing the optical image in Fig. 3 and resistance values in Fig. 4, we can speculate the electric conditions in each region of the graphene flake. For the area between electrodes 1 and 2, we obtained, $R_{(1,2)}=1.1 \mathrm{k} \Omega$ and $V_{\mathrm{CNP}(1,2)}=30.4 \mathrm{~V}\left(n_{(1,2)} \approx 2.12 \times 10^{12} \mathrm{~cm}^{-2}\right)$. Whereas area between electrodes 2 and 3 had both $R_{(2,3)}=1.24 \mathrm{k} \Omega$ and $V_{\mathrm{CNP}(2,3)}=40.4 \mathrm{~V}\left(n_{(2,3)} \approx 2.82 \times 10^{12} \mathrm{~cm}^{-2}\right)$ larger than that between electrodes 1 and 2 . Since the ratio of edge area on the graphene channel between electrodes 2 and 3 is larger than that between 1 and 2 , the edge contributes to the large resistance and hole-doping due to disorders and unremoved PMMA residues on the edge, respectively. On the other hand, although the channel area between electrode 3 and 4 has a similar edge ratio as that between electrode 1 and 2, the $R_{(3,4)}=0.7 \mathrm{k} \Omega$ and $V_{\mathrm{CNP}(3,4)}=38 \mathrm{~V} \mathrm{( \textrm {n } _ { ( 3 , 4 ) } \approx 2 . 6 6}$ $\times 10^{12} \mathrm{~cm}^{-2}$ ) are smaller and larger than $R_{(1,2)}$ and $V_{\mathrm{CNP}(1,2)}$, respectively. This manifests that although PMMA residues at the edge contribute equally to $n_{(1,2)}$ and $n_{(3,4)}$, the Au deposition (marked with A) allows to suppress $R_{(3,4)}$ and elevate $n_{(3,4)}$ compared with $R_{(1,2)}$ and $n_{(1,2)}$, respectively. When we inspected the full area between electrodes 1 and $4, R_{(1,4)}=1.58 \mathrm{k} \Omega$ and $V_{\mathrm{CNP}(1,4)}=32.5 \mathrm{~V}\left(n_{(1,4)} \approx 2.27 \times 10^{12} \mathrm{~cm}^{-2}\right)$ was the largest and similar as $\mathrm{n}_{(1,2)}$. Since the edge contribution is not dominant in the full area between electrodes 1 and $4, n_{(1,4)}$ is similar to the averaged values of $n_{(1,2)}, n_{(2,3)}$, and $n_{(3,4)}$. However, owing to the disorders, such as the folded area (marked by $\mathrm{B}), R_{(1,4)}$ exhibits the largest value. Consequently, by performing transport characterization of selected areas of graphene, the partial resistance and doping deviation in a graphene flake can be diagnosed.

\section{Conclusions}

In this work, we showed that the resistance and doping deviation 
in graphene depending on the position between the center area of the flake and the edge could be diagnosed by transport characterization. In particular, we observed that the edge leads to suppression of conductance and increased doping due to increasing disorders and PMMA residues, respectively. This result will help understand the origin of the difference in resistance and properties in different areas of graphene.

\section{Acknowledgments}

This work was supported by the research fund of Chungnam National University.

\section{References}

[1] K. S. Kim, Z. Yue, H. Jang, S. Y. Lee, J. M. Kim, K. S. Kim, J. H. Ahn, P. Kim, J. Y. Choi, and B. H. Hong, Nature 457, 706 (2009).

[2] X. Li, W. Cai, J. An, S. Kim, J. Nah, D. Yang, R. Piner, A. Velamakanni, I. Jung, E. Tutuc, S. K. Banerjee, L. Colombo, and L.
R. S. Ruoff, Science 324, 1312 (2009).

[3] S. Bae, H. Kim, Y. Lee, X. Xu, J. S. Park, Y. Zheng, J. Balakrishnan, T. Lei, H. R. Kim, and Y. I. Song, Nat. Nanotechnol. 5, 574 (2010).

[4] J. S. Choi, H. Choi, K. C. Kim, H. Y. Jeong, Y. J. Yu, J. T. Kim, J. S. Kim, and C. G. Choi, Sci. Rep. 6, 24525 (2016).

[5] J. H. Kim, M. M. Haidari, J. S. Choi, H. Kim, Y. J. Yu, and J. Park, J. Korean Phys. Soc. 72, 1045 (2018).

[6] W. Kim, K. Yoo, E. K. Seo, S. J. Kim, and C. Hwang, J. Korean Phys. Soc. 59, 71 (2011).

[7] J. Nam, I. Lee, D. Y. Lee, M. J. Kim, and K. S. Kim, J. Korean Phys. Soc. 76, 923 (2020).

[8] Y. J. Yu, Y. Zhao, S. Ryu, L. E. Brus, K. S. Kim, and P. Kim, Nano Lett. 9, 3430 (2009).

[9] Y. J. Yu, J. H. Choe, J. Y. Kim, O. H. Gwon, H. K. Choi, J. S. Choi, J. H. Kim, J. S. Kim, J. T. Kim, J. H. Shin, and Y. K. Choi, Nanoscale 11, 4753 (2019).

[10] Y. J. Yu, J. Korean Phys. Soc. 74, 132 (2019).

[11] Y. J. Yu, Appl. Sci. Converg. Technol. 28, 51 (2019).

[12] Y. J. Yu, J. Korean Phys. Soc. 76, 727 (2020). 